\title{
THE SELECTION OF BORON NITRIDE CIRCLES FOR GRINDING SAPONITE-TITANIUM COMPOSITES USING NON-PARAMETRIC METHOD
}

\author{
N.M. Huliieva*, D.O. Somov, V.V. Pasternak, L.M. Samchuk, T.I. Chetverzhuk \\ Lutsk National Technical University, \\ 75 Lvivska Str., 43018 Lutsk, UKRAINE \\ *e-mail: n.huliieva@Intu.edu.ua
}

The issue of grinding saponite-titanium composites has not been considered in the machine building industry yet. The reason is that the chips are stuck on the working surfaces of abrasive tools made of silicon carbide and electrocorundum. This is due to the high adhesive activity at operating cutting temperatures between the composite and traditional abrasives.

The article aims at studying the grinding of saponite-titanium composites using abrasive tools in various cutting modes based on parametric and non-parametric statistical methods.

To solve this problem, high porous wheels (HPW) made of cubic boron nitride CBN30 with $100 \%$ concentration on a bond V (K27), a pore-forming KF40, varied grains: B76, B126, B151 (ISO 6106:2013) - and hardness: M and O (ISO 525:2013) were used to grind saponitetitanium composites. Additionally, the Norton wheels from green silicon carbide with a normal porosity $39 \mathrm{C}(46 ; 60) \mathrm{K} 8 \mathrm{VK}$ and with different grain size were tested. Norton wheels provide reduction of roughness height by $1.4-1.5$ times in comparison with boron nitride HPW. These are recommended for the finishing grinding stage and HPW CBN30 - the preliminary to reduce the thermal effects on composites. By processing stability, the Norton wheels with grain 46 rank first, and among boron nitride HPW - CBN30 B76 100 OV K27-KF40.

Keywords: Abrasive tools, composite, grinding, parametric and nonparametric methods, saponite-titanium.

\section{INTRODUCTION}

Saponite-titanium composite is a promising material for mechanical engineering thanks to the well-designed manufacturing technology and relatively low cost [1]-[3]. It has unique physical and chemical properties and is one of the most difficult to pro- 
cess composite. Its properties make the cutting process difficult even when compared to processing materials such as cast iron and stainless steel. However, if you plan machining step-by-step using the necessary knowledge in the field and the appropriate tools and equipment, there is a possibility for the grinding process [4]-[14].

The goal of the study is to grind saponite-titanium composites using abrasive tools in various cutting modes.

The main objectives of the study are to select optimal equipment and tools, to choose the required cutting modes and to develop grinding techniques. The object of the study is finishing and fine grinding operations.The subject of the study is the regularities of the formation of quality parameters in the process of mechanical processing of fine grinding.

According to the results of the studies, it is established that when grinding saponite-titanium blanks traditional abrasives based on silicon carbide are used. This is due to the fact that saponite-titanium intensively gives electrons to aluminum atoms in corundum, causing adhesive wear of electrocorundum grains. Adhesion interaction with silicon carbide is less intense than with electrocorundum. The properties of the composite, which affect its interaction with abrasive materials, are reflected in their wear resistance. Thus, for saponitetitanium, the wear resistance of silicon carbide is twice as high relative to corundum materials [15]. Information on the choice of green silicon carbide grains is private and requires further study in each case. Abrasive materials whose atoms do not accept saponite-titanium electrons and thus minimize adhesion are cubic boron nitride (CBN) and diamond. Clogging of the working surface of the wheels is caused by the low antifriction parameters of the composite, which has low wear resistance and a high ten- dency to ignition during friction. The thin oxide film is easily destroyed because it is thinner than the inner material, due to the diffusion of hydrogen, oxygen and nitrogen at grinding temperatures, starting from $350 \ldots 550{ }^{\circ} \mathrm{C}$ [16]-[18]. The surface quality of saponite-titanium parts is evaluated by the surface roughness, which is one of the most important parameters in topography. They are formed by the following primary values:

$H=\sum_{m=1}^{5} h_{m} \sum_{m=1}^{5} h_{m}$,

where $h_{1} h_{1}$ is a component of the profile that reflects the kinematic transfer of the geometric characteristics of the relief of the working surface of the tool on the workpiece (mold); $h_{2} h_{2}$ is a component that arises as a result of oscillations of the circle and the workpiece due to different heights and chaotic arrangements of grains in the bundle; $h_{3} h_{3}$ is a component due to plastic deformation of the surface when cutting grains into the metal; $h_{4} h_{4}$ is a component of the adhesive interaction of grains with the workpiece; $h_{5} h_{5}$ is a component of elastic deformations of the circle.

According to authors [19], the dominant component is $h_{1} h_{1}$, and all others $h_{m}$ $h_{m}, h_{m} h_{m}=\overline{2 ; 52 ; 5}$ are secondary values. It is established that the radius of the cutting part of the grains depends not only on their material, but also on the depth of incision in the metal. As the depth increases, individual small scratches merge into large one [12]. The weakest link in the technological system of the grinding process is the abrasive circle. At the same time, in the considered publications the emphasis is put on other technological parameters and conditions of grinding, and circles apply without the 
necessary substantiation. For this reason, it has been decided to dwell on the choice of grain size of CBN HPC and Norton circles of standard porosity, as well as to further study the effect of hardness of CBN HPC on surface roughness. Given the sensitiv- ity of the saponite-titanium alloy to stress concentrators, we consider it appropriate to optimise the characteristics of the circles by the criterion of roughness of parts, taking into account the surface roughness.

\section{MATERIALS AND METHODS}

For the experiment, we used 3D711VF11, 3G71M flat-grinding machines [20] and abrasive circles for grinding. The shape, size and technological parameters are shown in Table 1.

Table 1. Basic Parameters of Grinding Circles

\begin{tabular}{|c|c|c|c|c|c|c|}
\hline \multirow[b]{2}{*}{ Circle } & \multirow[b]{2}{*}{$\begin{array}{l}\text { Shape and } \\
\text { dimensions }\end{array}$} & \multicolumn{5}{|c|}{ Technological parameters } \\
\hline & & $\begin{array}{c}\text { Speed, } V_{g}, \\
\mathrm{~m} / \mathrm{s}\end{array}$ & $\begin{array}{c}\text { Traverse, } S_{t}, \\
\mathrm{~m} / \mathrm{min}\end{array}$ & $\begin{array}{c}\text { Cross feed, } \\
S_{c}, \\
\mathrm{~mm} / \text { double } \\
\text { movement }\end{array}$ & $\begin{array}{c}\text { Cutting } \\
\text { depth, } t, \mathrm{~mm}\end{array}$ & $\begin{array}{c}\text { Operating } \\
\text { overmeasure, } \\
z, \mathrm{~mm}\end{array}$ \\
\hline HPC CBN & $1 \mathrm{~A} 1200 \times 20 \times 76 \times 5$ & 30 & \multirow{2}{*}{8} & \multirow{2}{*}{6} & \multirow{2}{*}{0.01} & \multirow{2}{*}{0.1} \\
\hline Norton & $01250 \times 20 \times 76$ & 35 & & & & \\
\hline
\end{tabular}

During the experiment, $S_{c}$ feed is selected for two passes, so it makes sense to explain the functional purpose. Lowering the table to the depth was performed when the longitudinal table was shifted to the leftmost position relative to the operator. In this regard, moving the table from left to right is considered working. Cutting of metal from the surface was carried out according to the counter-grinding scheme, since the circle had to rotate clockwise. Then the reverse movement of the table together with the workpiece within the specified feed of the $\mathrm{S}_{\mathrm{c}}$ became opposite. Given that the value of the $S_{c}$ is less than the height of the abrasive tool, the residual working surface of the circle provides processing of the surface of the longitudinal movement of the table in both directions. In this case, the finishing pass of the tool occurred in the conditions of passing grinding. The circle embedding scheme was chosen based on the results of research [19], in which saponite-titanium alloy billets were processed under deep (singlepass) grinding conditions and received a reduction in the roughness height by one categorical value (CV) [21] in comparison with the alternative counter-grinding scheme.

Samples of saponite-titanium were used for the experiment [1]. Their physical parameters are shown in Table 2. Grinding was performed on the plane N. Coolant $5 \%$ emulsion Aquol 11 (TU 38.101932-83) was supplied by irrigation to the part with a flow rate of $8 \ldots 121 / \mathrm{min}$.

Table 2. Physical Parameters of Experimental Samples

\begin{tabular}{|l|l|l|l|l|}
\hline Composite & Size, $\mathrm{D} \times \mathrm{h}, \mathrm{mm}$ & $\sigma, \mathrm{MPa}$ & $\delta, \%$ & $\mathrm{E}, \mathrm{GPa}$ \\
\hline Saponite-titanium & $3.5 \times 50$ & 700 & $6 \ldots 10$ & 60 \\
\hline
\end{tabular}


The number of duplicate experiments $-\mathrm{n}=20 ; \mathrm{i}=\overline{1 ; 20}$. Variable grinding conditions are represented by the code "dijv", which is convenient for analysing the initial process parameters using statistical methods. In this case, the index $d=1 ; 2$ shows the direction of the roughness arrangement: 1 is parallel to the vector $S_{c}, 2$ is parallel to the vector $\mathrm{S}_{\mathrm{t}}$. Circle characteristics are encoded by the index $\mathrm{i}=\overline{1 ; 6}$ : 1 is CBN30 B76 100 OV K27-KF40; 2 is CBN30 B126 100 MV K27-KF40; 3 is CBN30 B126 100 OV K27-KF40; 4 - CBN30 B151 100 OV $\mathrm{K} 27-\mathrm{KF} 40 ; 5$ is 39C $46 \mathrm{~K} 8 \mathrm{VK} ; 6-39 \mathrm{C} 60$ $\mathrm{K} 8 \mathrm{VK}$. HPC $\mathrm{i}=\overline{1 ;}$; refer to cubic boron nitride $(\mathrm{CBN})$ instruments [22], in which the grain size varies from B76 (200/250) to B203 (120/140) and hardness from M (average) to $\mathrm{O}$ (average hardness) [23]. Norton $\mathrm{i}=\overline{5 ; 6}$ circles of green silicon carbide grains of the 8th structure (normal porosity) have a medium hardness $(\mathrm{K})$ and differ in grain size: $46(355 \ldots 300 \mu \mathrm{m})$ and $60(300$ ... 250 $\mu \mathrm{m})$ according to ISO 8486-1:1999 [24], where the basic fraction are given in parentheses. Index $\mathrm{j}=\overline{1 ; 3}$ is used in the scattering coefficients: 1 is for $\mathrm{SD}_{\text {di }} ; 2$ is for $\mathrm{R}_{\mathrm{di}}$; 3 is for $\mathrm{QL}_{\mathrm{di}}$.

Surface roughness measurements were performed using a system based on a profilograph: profilometer model 252 of the plant "Caliber" [25]. The system is a contact device for sequential conversion of the profile type AI. The device is intended for measurement in laboratory languages of roughness and roughness of a surface of products from metal and nonmetallic products. Its technical characteristics are given in Table 3. The measurement is performed on a straight line of the cut plane.

Table 3. Technical Characteristics of the Profilograph: Profilometer Model 252

\begin{tabular}{|c|c|c|c|c|c|c|c|c|c|}
\hline \multicolumn{7}{|c|}{ Parameters } & \multirow{3}{*}{$\begin{array}{l}\text { Step compart- } \\
\text { ment value, mm }\end{array}$} & \multirow{3}{*}{$\begin{array}{c}\text { Type of } \\
\text { cutting } \\
\text { agent }\end{array}$} & \multirow{3}{*}{$\mathrm{m}, \mathrm{kg}$} \\
\hline \multicolumn{2}{|c|}{ profilograph } & \multicolumn{5}{|c|}{ profilometer } & & & \\
\hline $\mathrm{R}_{\mathrm{z}}, \mu \mathrm{m}$ & $\begin{array}{c}1, \\
\mathrm{~mm}\end{array}$ & $\mathrm{R}_{\mathrm{a}}, \mu \mathrm{m}$ & $\begin{array}{c}\mathrm{H}_{\min }, \mathrm{H}_{\max }, \\
\mu \mathrm{m}\end{array}$ & tp, $\%$ & $\mathrm{n}$ & $1, \mathrm{~mm}$ & & & \\
\hline $0.02-250$ & 50 & $0.02-100$ & $0.1-100$ & $0-100$ & up to 1000 & $1.5 ; 3 ; 6$ & $0.08 ; 0.25 ; 0.8 ; 2.5$ & numbers & 107.6 \\
\hline
\end{tabular}

Profilograph: profilometer model 252 consists of a rack with a motor drive, a universal subject table, a control unit, a computing unit, a differential inductive measuring transducer with a diamond probe needle and a recording device.

During experimental studies, according to ISO 1302:2002 [21], the following parameters $R_{a}, R_{q}, R_{z}, R_{\max I}$ were determined related to the height properties of inequalities. $R_{a}$ is the arithmetic mean deviation of the profile; $R_{q}$ is the standard deviation of the profile; $R_{z}$ is the height of the profile irregularities at ten points; $R_{\max }$ is the largest height of profile irregularities. Surface roughness has both technical and economic tasks. The main parameters are the requirements for accuracy, efficiency, reliability of parts and equipment of machines. They depend on many roughness parameters, mechanical characteristics of surface functionality and method of application. The main requirement is to improve the service life and performance of engineering products.

The arithmetic mean deviation of the profile $R_{a}$ is the arithmetic mean of the absolute values of the deviations of the profile within the base length:

$R_{a}=\frac{1}{l} \cdot \int_{0}^{l}|y(x)| \cdot d x$,

where $y$ is the deviation of the profile: the distance between any point of the profile and the center line, which is measured along the normal line drawn to the center 
line through this point of the profile;

$l$ is the base length.

The standard deviation of the profile $R_{q}$ within the base length:

$$
R_{q}=\sqrt{\frac{1}{l} \int_{0}^{l} y^{2}(x) d x}
$$

The height of the irregularities of the profile at ten points $R_{z}$ is the sum of the average absolute values of the heights of the five largest protrusions of the profile and the depths of the five largest depressions of the profile within the base length:

$R_{Z}=\frac{\sum_{i=1}^{5}\left|y_{p i}\right|+\sum_{i=1}^{5}\left|y_{v i}\right|}{5}$,

where $y_{p i}$ is the height of the $i$ largest protrusion of the profile;

$y_{v i}$ is the depth of the $i$ largest depression of the profile.

The maximum height of the irregularities of the profile $R_{\max }$ is the distance between the line of protrusions and the line of depressions of the profile within the base length.

In this paper, we consider the parameters of $R_{a}, R_{\max }$, which are the most important for saponite-titanium composites, which primarily affect the quality and performance of manufactured parts.

Methods of statistical interpretation of experimental data. Taking into account the instability of the grinding process and the random formation of the roughness surface, the analysis of observations is carried out using statistical approaches. We consider them as random variables (RV) that form independent sets:

$$
\left\{y_{i v}\right\}=\overline{1 ; 6}, v=\overline{1 ; 20} \text {. }
$$

In technical applications, we use parametric and non-parametric statistical meth- ods (for example, rank methods). Characteristics of one-dimensional frequency distribution for many (2) are [26]: where $\bar{y}_{i}=y_{i}$ for the first direction - the medium, $\mathrm{SD}_{\mathrm{i}}-$ deviation standards, $R_{i}=\left|y_{\max }-y_{\text {min }}\right|$ $\left|y_{\max }-y_{\text {min }}\right|_{i-}$ scopes, $\tilde{y}_{i}-$ for the second direction - medians, $Q L_{\mathrm{il}}=\left|y_{0,75}-y_{0,25}\right|_{i}-$ quartile latitudes, covering $50 \%$ of the majority of observations (2). The first frequency characterises the position measure (total value), the next - the scattering boundaries (precision). $A_{S i}=\left[\frac{3(y \cdot-\tilde{y})}{S D}\right]_{i}-$ shift irelative to $\mathrm{yi} \cdot$ due to the asymmetry (curvature) of the distribution curves found from the expression.

Each method of statistics has its own area of rational application. For the parametric method, it is necessary that most of the values (2) satisfy the requirements of normality and homoscedasticity of the dispersion. The second restriction is that measurements (2) must be determined with high accuracy, because otherwise it may lead to the adoption of incorrect hypotheses. To check the obtained data, one must use a non-parametric method that is not related to the properties of random variables.

Performing a one-dimensional variance analysis (OVA) and multiple search for predicted values $\left(\bar{y}_{m y}\right)_{i}, \mathrm{i}=\overline{1 ; 6}$ is associated with large calculations, so we use the program Statistica 12 [27].

The technique of modelling fuzzy logic. Fuzzy logic is designed to create mathematical models based on linguistic reasoning, in which the language and experience of experts play a fundamental role. In this sense, fuzzy logic is equivalent to the theory of fuzzy sets, i.e., classes with inaccurate, blurred boundaries. The theory of fuzzy sets [28] is a generalization and rethinking of the most important areas of classical mathematics. Moreover, by fuzzy sets $A_{i l}$ we mean sets of ordered pairs composed of elements yilv of universal sets $\{y i l v\}$ and 
the corresponding degrees of membership $\mu$ $\{y i l v\}$ :

$A_{i l}=\left\{\left(y_{i l v}, \mu_{A}\left(y_{i l v}\right)\right) ; y_{i l v} \in\left\{y_{i l v}\right\}\right\}$,

where $\mu_{A}\left(y_{i l v}\right)$ are characteristic functions indicating the degree to which yilv belongs to fuzzy sets $A_{i l}$. When implementing the process of modeling fuzzy logic, attributes are created that are based simultaneously on measures of position and scattering, which is impossible for statistical methods.

\section{EXPERIMENTAL RESULTS AND DISCUSSION}

Data (2) were tested for uniformity of variances (null hypotheses $\mathrm{H}_{0}$ ) for eight roughness parameters in two directions $\mathrm{d}=\overline{1 ; 2}$ when working with circles $\mathrm{i}=$ $\overline{1 ; 6}$, for which three groups of criteria were used $(\mathrm{w}=\overline{1 ; 3})$ : 1 - Hartley, Cochran, Bartlett, 2 - Levene, 3 - Brown-Forsyth. According to the test results, $\mathrm{H}_{0}$ was accepted if the majority of decisions in its favour were $f_{0} \in[19]$. It is established that for roughnesses $\left(R_{a}, R_{q}, R_{z}, R_{\max }\right)_{2}$ in the longitudinal direction $\mathrm{H}_{0}$ is accepted at $\mathrm{f}_{0} \in[29]$. In the orthogonal direction $\mathrm{d}=1$ for the first group of criteria $\mathrm{w}=1$, all $\mathrm{H}_{0}$ are accepted with a minor error of the $2^{\text {nd }}$ kind. According to other statistical indicators $\mathrm{w}=2,3$, the homogeneity of variances was confirmed for the parameters $\mathrm{R}_{\mathrm{a} 1}(\mathrm{w}=2$; $3)$ and $R_{q 1}(w=3)$ in the presence of an error of the $2^{\text {nd }}$ kind. Hypotheses about the normality of data distributions (2) behind the circles $i=\overline{1 ; 6}$ and the roughness parameters are accepted by the Shapiro-Wilk criterion when performing inequalities: $\alpha>0.5$. The test results are shown in Table 4.

Table 4. Normality of Data Distributions (2) for Circles $i=(\overline{1 ; 6})$ and Roughness Parameters

\begin{tabular}{|c|c|c|c|c|c|c|c|}
\hline \multicolumn{8}{|c|}{ Circle $\mathrm{i}=\overline{1 ; 6}$} \\
\hline & & 1 & 2 & 3 & 4 & 5 & 6 \\
\hline \multirow{8}{*}{$\alpha_{i}, \mu \mathrm{m}$} & $\mathrm{R}_{\mathrm{a}}$ & 0.2986 & 0.2147 & 0.6014 & 0.2425 & 0.0916 & 0.7755 \\
\hline & $\mathrm{R}_{\mathrm{gl}}$ & 0.8765 & 0.4166 & 0.4241 & 0.1227 & 0.1137 & 0.8114 \\
\hline & $\mathrm{R}_{71}$ & 0.5735 & 0.8297 & 0.3391 & 0.0241 & 0.0982 & 0.9432 \\
\hline & $\mathrm{R}_{\max 1}$ & 0.0009 & 0.8635 & 0.0047 & 0.0047 & 0.4036 & 0.3355 \\
\hline & $\mathrm{R}_{\mathrm{a}_{2}}$ & 0.395 & 0.9493 & 0.047 & 0.1238 & 0.000002 & 0.000007 \\
\hline & $\mathrm{R}_{0}$ & 0.7233 & 0.6187 & 0.1249 & 0.1397 & 0.000001 & 0.000004 \\
\hline & $\mathrm{R}_{72}$ & 0.2472 & 0.9878 & 0.0112 & 0.0354 & 0.0002 & 0.00002 \\
\hline & $\mathrm{R}_{\max 2}$ & 0.6004 & 0.7021 & 0.3524 & 0.2747 & 0.00001 & 0.00002 \\
\hline
\end{tabular}

As can be seen from Table 4, the best results of the normality of the distributions were obtained by grinding the details of saponite-titanium nitriding HPC B126 with a hardness $M(i=2)$ : from eight parameters $\mathrm{H}_{0}$ we accept six. Given that the roughness parameters in the $\mathrm{d}=1$ direction exceed the longitudinal counterparts and prevail in ensuring the operational properties of the parts [21], the priorities between the circles should be redistributed. According to the indicators, the first position falls on the circle $39 \mathrm{C}(\mathrm{i}=6)$ with a grain size of 60 , for which the normality of distributions throughout the transverse parameter is ensured. At the next position CBN HPC $\mathrm{i}=1 ; 2$. As we can see, the requirements for $\mathrm{RV}$ require that the parametric method is not fully met. There is a need to apply the priority direction of interpretation of exper- 
imental data of rank statistics.

Evaluation of cutting properties circles under the provision of the move. At the first stage of the analysis of experimental data, we will clarify their relationship in two orthogonal directions. According to the experimental medians

$\left(\frac{\tilde{y}_{1}}{\tilde{y}_{2}}\right)_{i}, i=\overline{1 ; 6}$,

it is established that for some roughness parameters, the ratios are:

$$
\begin{aligned}
& \left(\frac{\tilde{R}_{a 1}}{\tilde{R} a_{2}}\right)_{i}=2,11-3,02, \\
& \left(\frac{\tilde{R} q_{q 1}}{\tilde{R} q_{2}}\right)_{i}=2,14-3,02, \\
& \left(\frac{\tilde{R}_{z 1}}{\tilde{R} z_{2}}\right)_{i}=2,35-3,1, \\
& \left(\frac{\tilde{R}_{\max 1}}{\tilde{R}_{\max 2}}\right)_{i}=2,04-2,71 .
\end{aligned}
$$

The smallest anisotropy of the tool roughness is provided when grinding the nitroborne HPC CBN30 B126 100 MV K27-KF40, and the largest with a Norton grain size of $46(i=5)$. According to the roughness parameters, the smallest isotropy of the topography refers to $R_{z}$ and the highest to $\mathrm{R}_{\max }$. Anisotropy of roughness $d=1 ; 2$ should be used to enhance the performance of the parts, positioning them during grinding so that longitudinal roughness receives the highest load when operating the machines. When operating the machines, the greatest information $\left(\mathrm{R}_{\mathrm{a} 1}\right.$, $\mathrm{R}_{\max }$ ) is located in the transverse direction.

The obtained experimental and expected maximum values will be analysed in two aspects: from the standpoint of statistics and grinding technology.

In the first case, it was found that for parameters $R_{a}, R_{q}, R_{z}, R_{\text {maxl }}$, which were partially represented (Tables 5 and 6), twenty research medians $\tilde{y}_{l i}, \mathrm{i}=\overline{1 ; 6}$ out of the total number $\mathrm{N}=4 \times 6=24$ were smaller than the same research averages. However, for only one roughness $R_{a 12}$, the parametric position measure exceeded the median by one RV. For the rest of the roughness range, the error $\tilde{y}_{l i}$ relative to $\tilde{y}_{l i}$ occurred within the RV. A situation, in which the median coefficients (5) were less than one, revealed additional reserves for improving machine performance or grinding performance. The results obtained indicate that it is advisable to use the rank method instead of the Gaussian method to the competitor, which "for other results" [29] showed less accuracy in searching for common values.

Table 5. Influence of Characteristics, Roughness Position and Coefficients (8)-(11) for Parameters $R_{a l i}$

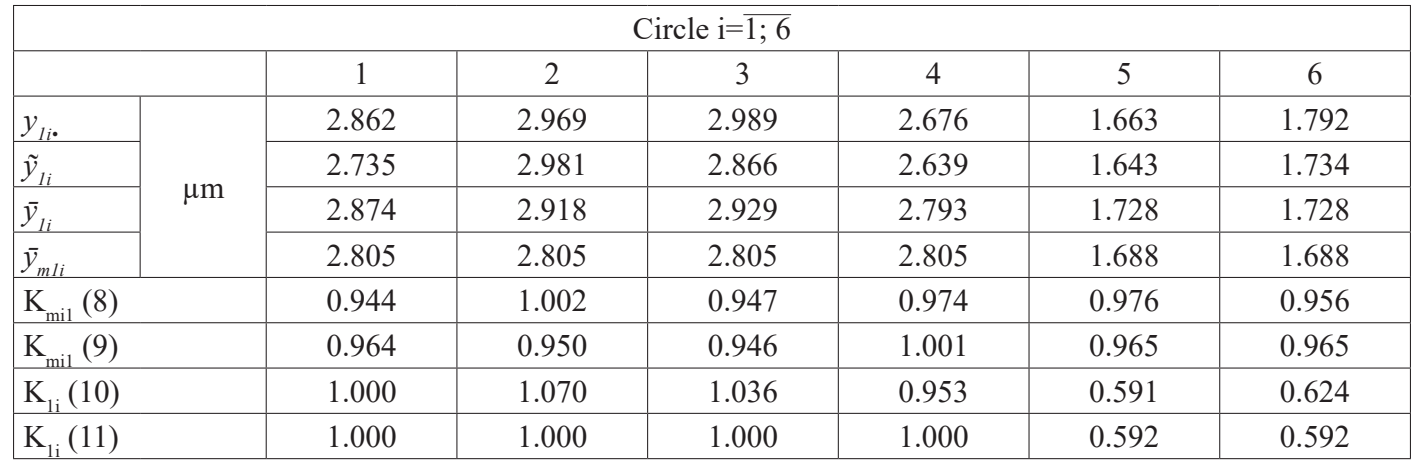


Table 6. Influence of Characteristics, Roughness Position and Coefficients (8)-(11) for Parameters $R_{\max l i}$

\begin{tabular}{|c|c|c|c|c|c|c|c|}
\hline \multicolumn{8}{|c|}{ Circle $i=\overline{1 ; 6}$} \\
\hline & & 1 & 2 & 3 & 4 & 5 & 6 \\
\hline $\mathrm{y}_{1 \mathrm{i}}$ & \multirow{4}{*}{$\mu \mathrm{m}$} & 2.862 & 2.969 & 2.989 & 2.676 & 1.663 & 1.792 \\
\hline$\tilde{y}_{1 i}$ & & 2.735 & 2.981 & 2.866 & 2.639 & 1.643 & 1.734 \\
\hline$\overline{\mathrm{y}}_{1 \mathrm{i}}$ & & 2.874 & 2.918 & 2.929 & 2.793 & 1.728 & 1.728 \\
\hline$\overline{\mathrm{y}}_{\mathrm{mli}}$ & & 2.805 & 2.805 & 2.805 & 2.805 & 1.688 & 1.688 \\
\hline \multicolumn{2}{|c|}{$\mathrm{K}_{\text {mil }}(8)$} & 0.944 & 1.002 & 0.947 & 0.974 & 0.976 & 0.956 \\
\hline \multicolumn{2}{|c|}{$\mathrm{K}_{\text {mil }}(9)$} & 0.964 & 0.950 & 0.946 & 1.001 & 0.965 & 0.965 \\
\hline \multicolumn{2}{|c|}{$\mathrm{K}_{1 \mathrm{i}}(10)$} & 1.000 & 1.070 & 1.036 & 0.953 & 0.591 & 0.624 \\
\hline \multicolumn{2}{|c|}{$\mathrm{K}_{1 \mathrm{i}}(11)$} & 1.000 & 1.000 & 1.000 & 1.000 & 0.592 & 0.592 \\
\hline
\end{tabular}

Differences between the media coefficients (8) and (9) also confirm the need for the second stage of the OVA. The results are the same when evaluating the cutting properties of circles relative to formulas (10) and (11). The fact is that for the experienced medians (10), the cutting abilities of the circles $i=1 ; 4$ differ from each other, and for the expected analogues $y_{m l i}=\overline{2 ; 4}$ the coefficients are equal to one, including for the roughness $z_{l}\left(R_{q}, R_{z}\right)_{l i}$. Thus, for boron nitride HPC $i=1 ; 4$ grain varies from B76 to $\mathrm{B} 151$ and a decrease in the degree of hardness from $\mathrm{O}$ to $\mathrm{M}$ at $5 \%$ level, which is considered insignificant. This is most likely due to the modulus of elasticity of saponite-titanium billets, which is almost one and a half times lower in comparison with steel. With an increase in the cutting temperature to $350{ }^{\circ} \mathrm{C}$, it additionally decreases almost linearly [30], which increases the elasticity after the action of the composite, which leads to an increase in the cutting force on the inner surfaces of the grains. It strengthens the elastic tension in the technological link "blank-HPC", acting as a vibration damper [31]. These regularities are accompanied by a decrease in the components $h_{2}$ and $h_{5}$ in Eq. (5). Additionally, the elementary values of $h_{3}$ and $h_{4}$, respectively, are reduced due to the low plasticity of the saponite-titanium alloy and the high chemical inertness of CBN grains in relation to composite. However, $\mathrm{h}_{3}$ we believe that when grinding, the cutting temperature is from $500 \ldots 600^{\circ} \mathrm{C}$ or higher. This in turn can cause a significant reduction in strength and fluidity of saponite-titanium composites with improved ductility, as specified in [1] by continuous heating of samples and due to the fact that the temperature of the grinding surface is characterised by a high gradient of decrease. Additionally, the elementary values of $h_{3} i h_{4}$, respectively, are reduced due to the low plasticity of the composite alloy and the high chemical inertness of $\mathrm{CBN}$ grains in relation to saponite-titanium.

According to the results of the research, the properties of grinding parts of saponite-titanium are most acceptable for the samples obtained under similar conditions for high-speed plates (HSP) B9Mo4Co8 and blanks made of steel $06 \mathrm{Cr} 14 \mathrm{Ni} 6 \mathrm{Cu}-$ 2MoWT. It was found that when grinding the HSP, the smoothest surface was provided for the B126 grain, which was less than two CV for B76 and B151. At the same time, the increase in the HPC hardness $i=$ $2 ; 3$ in the specified interval was accompanied by a decrease in the reference values by two $\mathrm{CV}$. At the same time, when grinding $06 \mathrm{Cr} 14 \mathrm{Ni} 6 \mathrm{Cu} 2 \mathrm{MoWT}$ blanks, the role of selecting the HPC hardness increased: the position boundaries for different roughness parameters decreased by $2 \ldots 3 \mathrm{CV}$. At the same time, the minimum roughness is reached at a grain size of $\mathrm{B} 151$. The results 
obtained indicate that the modulus of elasticity of steel billets increases almost twice as compared to saponite-titanium ones. This increases the influence of CBN grain sizes and the hardness of CBN HPC on the formation of general roughness values. It was revealed that the recommendations for the choice of saponite-titanium, B9Mo4Co8, 06Cr14Ni6Cu2MoWT were different. As previously stated, the roughness (1) is affected by the value $h 1$, and the rest are considered secondary values. However, the results of the studies showed significant differences in roughness at a constant component of $h_{1}$.

The cutting properties of Norton circles with a grit of 46 and 60 are provided for the expected medians of equivalence. Norton tools can reduce the roughness of CBN HPC by $1.5 \ldots 1.54$ times or by $3 \mathrm{CV}$. The results obtained allow concluding that it is advisable to perform rough grinding of the CBN HPC in order to minimise the thermal impact on the part [32] and finishing with Norton circles for effective reduction of micro irregularities.

When processing the batch of workpieces on the configured machines, the stability (reproducibility) of the process of grinding, which is regulated by scattering, plays a high role. Tables 7 and 8 present all three precision parameters $(\mathrm{SD}, \mathrm{R}, \mathrm{QL})_{1 \mathrm{i}}$.

In the case of non-parametric method, preference is given to the results predicted by $\mathrm{QL}_{1 \mathrm{i}}$ and coefficients (13). It was found that the variation of the granularity of the
CBN HPC from B76 to B151 at a constant degree of hardness $\mathrm{O}$ was approximated by an extreme curve with a maximum of $\mathrm{QL}_{13}$ with grain B126. At the same time, the minimum dependence of the QL $=\psi$ (graininess) for the parameters $R_{a 14}, R_{\max 14}, R_{q_{14}}$ was reached when grinding coarse-grained HPC B151 ( $\mathrm{i}=4)$, and for the height of the irregularities of the profile - at grain B76 $(\mathrm{i}=1)$.

For comparison, in the processing of blanks 06Cr14Ni6Cu2MoWT minimum QL is noted at the lowest grain size B76, and $\mathrm{QL}_{\max }$ - at $\mathrm{B} 126$ for all roughness parameters. The latter figure completely coincided with the results of grinding of saponite-titanium parts. In the HPC $\mathrm{i}=2$; 3 only the degree of hardness varies from medium $(i=2)$ to non-solid medium $(i=$ $3)$. The only correlation relationship with QL was not found between them: $\mathrm{QL}_{12}=$ $\mathrm{QL}_{13}$ - for parameter $\mathrm{R}_{\mathrm{a}} ; \mathrm{QL}_{12}<\mathrm{QL}_{13}-$ for the highest profile height. Another situation was for the details of $06 \mathrm{Cr} 14 \mathrm{Ni} 6 \mathrm{Cu}-$ 2MoWT: the increase in the hardness of the HPC in the studied range caused a decrease in the scattering rate by parameters $\mathrm{R}_{\mathrm{a} 1}$ and $\mathrm{R}_{\max 1}$ by 2.5 and 2.3 times, respectively. The parametric precision estimates for the saponite-titanium parts are predicted to be more stable and positioned the HPC in the following increasing sequence of scattering measures: 1 - B76, 2 - B151, 3 - B126M, $4-\mathrm{B} 126 \mathrm{O}$. The minimum and maximum granularity precision for both methods of statistics coincided (see Tables 7 and 8).

Table 7. Selective Estimates of the Cutting Properties of Circles of Scattering Boundaries $R_{a I i}$

\begin{tabular}{|l|c|c|c|c|c|c|c|}
\hline \multicolumn{2}{|c|}{} & 1 & 2 & 3 & 4 & 5 & 6 \\
\hline \multirow{2}{*}{$\mathrm{SD}_{1 \mathrm{i}}$} & \multirow{3}{*}{$\mu \mathrm{m}$} & 0.041 & 0.057 & 0.068 & 0.050 & 0.040 & 0.038 \\
\cline { 1 - 7 } $\mathrm{R}_{1 \mathrm{i}}$ & 0.245 & 0.299 & 0.358 & 0.274 & 0.252 & 0.183 \\
\cline { 3 - 8 } $\mathrm{QW}$ & & 0.048 & 0.078 & 0.078 & 0.052 & 0.043 & 0.075 \\
\hline \multirow{3}{*}{$\mathrm{K}_{1 \mathrm{ij}}$} & $\mathrm{j}=1(12)$ & 1.000 & 0.757 & 0.349 & 0.845 & 1.013 & 1.050 \\
\cline { 2 - 8 } & $\mathrm{j}=2(13)$ & 1.000 & 0.814 & 0.682 & 0.886 & 0.961 & 1.307 \\
\cline { 2 - 8 } & $\mathrm{j}=3(14)$ & 1.000 & 0.655 & 0.655 & 0.926 & 1.079 & 0.678 \\
\hline
\end{tabular}


Table 8. Selective Estimates of the Cutting Properties of Circles of Scattering Boundaries $R_{\max l i}$

\begin{tabular}{|c|c|c|c|c|c|c|c|}
\hline \multicolumn{8}{|c|}{ Circle $\mathrm{i}=\overline{1 ; 6}$} \\
\hline & & 1 & 2 & 3 & 4 & 5 & 6 \\
\hline $\mathrm{SD}_{\mathrm{li}}$ & \multirow{3}{*}{$\mu \mathrm{m}$} & 0.282 & 0.396 & 0.622 & 0.322 & 0.261 & 0.268 \\
\hline $\mathrm{R}_{\mathrm{l}}$ & & 1.142 & 1.882 & 2.659 & 1.265 & 1.218 & 1.086 \\
\hline $\mathrm{QW}_{1 \mathrm{i}}$ & & 1.000 & 0.560 & 0.582 & 0.401 & 0.375 & 0.450 \\
\hline \multirow{3}{*}{$\mathrm{K}_{\mathrm{lij}}$} & $\mathrm{j}=1(9)$ & 1.000 & 0.712 & 0.451 & 0.868 & 0.892 & 1.029 \\
\hline & $\mathrm{j}=2(10)$ & 1.000 & 0.597 & 0.420 & 0.892 & 0.924 & 1.039 \\
\hline & $\mathrm{j}=3(11)$ & 1.000 & 0.740 & 0.712 & 1.029 & 1.099 & 0.919 \\
\hline
\end{tabular}

For $(\mathrm{SD}, \mathrm{R})_{1 \mathrm{i}}, \mathrm{i}=2 ; 3$ unambiguous results were obtained, indicating a decrease in the reproducibility of the grinding process with increasing the hardness of the HPC from M to O: $1.16 \ldots 1.56$ times SD and $1.13 \ldots 1.41$ times the magnitude. In both cases, the greatest reduction in the stability of the grinding process was provided for the parameter $\mathrm{R}_{\max 1}$. As it was revealed before, at grinding of details of HPC observations (5) were characterised by a high degree of homogeneity of dispersions, and the rank method of their interpretation was chosen as a result of disturbances of normality of distributions (Table 1). Probably, for this reason, the results obtained are quite stable when evaluated by parametric scattering. It should be noted that increasing the stability of the grinding process by varying the hardness of the HPC must be carried out taking into account the material of the grinding parts. In particular, for saponitetitanium it is advisable to reduce, and for 06Cr14Ni6Cu2MoWT, on the contrary, to increase.

For Norton circles, the greatest stability is $1 \mathrm{QL}_{1 \mathrm{i}}, \mathrm{i}=5 ; 6$ and is shown when using larger grains 45 . This characteristic was even higher than for the basic HPC $\mathrm{i}=1$, as evidenced by the coefficients (14): 1.079 ... 1.099. Parametric estimates of the scattering boundaries of Norton circles, especially on a scale, for most grinding cases showed an increase in the reproducibility of processing when using Norton circles with smaller grains (at a grain size of 60).

\section{CONCLUSIONS}

1. In terms of violations of homogeneity of variances and uniform distributions, a nonparametric method is widely used in technical applications. The Gaussian competitor turned out to be expedient, in particular, to search for event position. It has been established that the average medians decrease, although this phenomenon occurs within the framework of the CV. This is evidenced by the median coefficients (8) and (9), which are less than one, in most cases. This, in turn, allows increasing the grinding performance while maintaining the surface quality of the parts.
2. It has been found that when grinding parts made of saponite-titanium composite, Norton circles provide high quality surface treatment in comparison with the $\mathrm{CBN}$ HPC. According to the research results, the median coefficients differ by $1.4 \ldots 1.5$ times. For this reason, the HPC CBN30 should be used for rough grinding to reduce the thermal impact of the circle on the part, and the Norton circle should be used for finishing grinding, where there are high requirements for the roughness of parts. Saponite-titanium composites have been found to be almost insensitive to variations 
in the grain size and hardness of the circles. This is due to the low modulus of elasticity of the saponite-titanium alloy.

3. The dispersion range under variable grinding conditions is more variable compared to the median and average. When using CBN HPC, the smallest $\mathrm{QL}_{1 \mathrm{i}}$ is provided for $\mathrm{i}=1 ; 4$ (that is, for grains B76 and B151). For B126 grain, the stability of the $\mathrm{QL}_{13}$ process decreased by $1.2 \ldots 1.3$ times. The variation in the HPC hardness from medium-hard $(i=3)$ to medium $(i=$ 2 ) should be recognised as insignificant QL. Simultaneously, the $(\mathrm{SD} \mathrm{R})_{1 \mathrm{i}} \mathrm{i}=2 ; 3$ shows an increase in the precision of the process. Therefore, at a high level of uniformity of variances, parametric estimates are more accurate than quartile latitudes.

\section{REFERENCES}

1. Guliieva, N. M. (2015). Getting Porous Penetrating Materials Using Natural Mineral - Saponite in Self-Propagating High-Temperature Synthesis. - Manuscript. Dissertation for the degree of Ph.D., specialty 05.02.01 - Materials science. Lutsk National Technical University, 134.

2. Rud', V. D., Samchuk, L. M., \& Gulieva, N. M. (2013). Using SHS process to obtain composite materials. Powder Metallurgy: Surface Engineering, New Powder Composite Materials. Welding: Collection Doc, 496-500.

3. Gulieva, N. M. (2014). Khimichnyi analiz ta fizychni vlastyvosti pryrodnogo material saponitu. Mizhvu zivskyi zbirnyk "Naukovi notatky", 44, 78-82.

4. Kar, S., Kumar, A S., Bandyopadhyay, P. P., \& Paul, S. (2020). Grindability of Plasma Sprayed Thermal Barrier Coating Using Super Abrasive Wheel. Transactions of the Institute of Metal Finishing, 98 (1), 30-36. DOI: 10.1080/00202967.2020.1697572.

5. Cao, J., Wu, Y., Li, J., \& Zhang, Q. (2015). A Grinding Force Model for Ultrasonic Assisted Internal Grinding (UAIG) of $\mathrm{SiC}$ Ceramics. Adv. Manuf. Technol., 81 (5-8), 875-885. DOI: 10.1007/s00170-015-72820 .

6. El Wakil, S. D., \& Srinagesh, K. (2008). Effect of the physical and mechanical properties of composites on their grinding characteristics. In Conf. HPSM 2008. April 2008 (pp. 149-155). WIT Transactions on the Built Environment 97: Grinding of polymeric composites. DOI: 10.2495/
HPSM080161.

7. Bianchi, E. C., Rodriguez, R. L., Hildebrandt, R. A., Lopes, J. C., de Mello, H. J., da Silva, R. B., \& de Aguiar, P. R. (2018). Plunge Cylindrical Grinding with the Minimum Quantity Lubrication Coolant Technique Assisted with Wheel Cleaning System. Manuf Technol, 95, 2907-2916. DOI: 10.1007/s00170-017-1396-5.

8. Wang, H., Ning, F., Hu, Yi., Fernando, P. K. S. C., Pei, Z. J., \& Cong, W. (2002). Surface Grinding of Carbon Fiber - Reinforced Plastic Composites Using Rotary Ultrasonic Machining: Effects of Tool Variables. Advances in Mechanical Engineering, 8 (9). DOI: 10.1177/1687814016670284.

9. Irani, R. A., Bauer, R. J., \& Warkentin, A. (2005). A Review of Cutting Fluid Application in the Grinding Process. Int $J$ Mach Tools Manuf, 45 (15), 1696-1705. DOI:10.1016/j.ijmachtools.2005.03.006.

10. Miyakawa, O., Watanabe, K., Okawa, S., Nakano, S., Shiokawa, N., Kobayashi, M., \& Tamura, H. (1990). Grinding of Titanium. 1. Commercial and Experimental Wheels Made of Silicon Carbide Abrasives. Shika Zairyo Kikai, 9 (1), 30-41. PMID: 2134811.

11. Jha, A., \& Paul, S. (2019).Surface Integrity in Grinding of Ti-6Al-4V Using Monolayer Superabrasive Wheel. Advances in Materials and Processing Technologies, 5 (2), 213-225. DOI: 10.1080/2374068X.2018.1564866.

12. Das, P., Bandyopadhyay, P. P., \& Paul., S. (2019). Finish Form Grinding of Thermally Sprayed Nano-Structured 
Coatings. Advances in Materials and Processing Technologies, 5 (1), 39-52. DOI: 10.1080/2374068X.2018.1510680

13. Paul, S, Singh, A. K., \&Ghosh, A. (2017). Grinding of Ti-6Al-4V under Small Quantity Cooling Lubrication Environment Using Alumina and MWCNT Nanofluids. Materials and Manufacturing Processes, 32 (6), 608-6015. DOI: 10.1080/10426914.2016.1257797

14. Li, H. N. (2016). Textured Grinding Wheels: A Review. International Journal of Machine Tools and Manufacture, 109, 8-35. DOI: 10.1016/j.ijmachtools.2016.07.001

15. Kacalak, W., Lipiński, D., Bałasz, B., Rypina, Ł., Tandecka, K., \& Szafraniec, F. (2018). Performance Evaluation of the Grinding Wheel with Aggregates of Grains in Grinding of Ti-6Al-4V Titanium Alloy. Advanced Manufacturing Technology, 94 (1-4), 301-314. DOI: 10.1007/s00170-0170905-x

16. Kosmac, T., Oblak, C., Jevnikar, P., Funduk, N., \& Marion, L. (1999). The Effect of Surface Grinding and Sandblasting on Flexural Strength and Reliability of Y-TZP Zirconia Ceramic. Dental Materials, 15 (6), 426-433. DOI: 10.1016/s0109-5641(99)00070-6

17. LópezdeLacalle, L. N., Rodríguez, A., Lamikiz, A., Celaya, A., \& Alberdi, R. (2011). Five-Axis Machining and Burnishing of Complex Parts for the Improvement of Surface Roughness. Materials and Manufacturing Processes, 26 (8), 997-1003. DOI: $10.1080 / 10426914.2010 .529589$.

18. Niharika, Agrawal, B.P., Khan, I.A., \& Khan, Z.A. (2016). Effects of Cutting Parameters on Quality of Surface Produced by Machining of Titanium Alloy and Their Optimization. CC BY 4.0, 63, 531-548. DOI: 10.1515/meceng-2016-0030.

19. Soler, Ya. I., \& Mai, D. S. (2015). Select of Abrasive Wheels while Pendular Grinding of Parts from Titanium Alloy VT22 by High Roughness Parameters. Equipment. Tools, 4 (69), 18-33. DOI: 10.17212/1994-63092015-4-18-30

20. Catalog (2020). Metal cutting machines. Catalog of metal-cutting machines and forging equipment. Rubicon LLC. Available at http://stanki-katalog.ru/sprav.htm

21. ISO (2002). ISO 1302:2002, Geometrical Product Specifications (GPS) - Indication of Surface Texture in Technical Product Documentation.

22. ISO (2013). ISO 6106:2013, Abrasive Products - Checking the Grain Size of Superabrasives.

23. ISO (2013). ISO 525:2013, Bonded Abrasive Products - General Requirements.

24. ISO (1996). ISO 8486-1:1996, Bonded Abrasives - Determination and Designation of Grain Size Distribution - Part 1. Macrogrits F4 to F220.

25. ISO (1987). ISO GOST 19300-86. Instruments for Measurement of Surface Roughness by the Profile Method. Contact Profilographs and Profilometers. Types and Main Parameters. Available at http://docs. cntd.ru/document/1200004988.

26. Jiang, J., Roussas, G. G., \& Samaniego, F. J. (2011). Nonparametric Statistical Methods and Related Topics. World Scientific, 480. DOI: $10.1142 / 8258$.

27. Statistica: Enterprise Capabilities, StatSoft, 22. Available at http://statsoft.ru/\#tabSTATIS

28. Ali, Y. M., \& Zhang, L. C. (2004). A Fuzzy Model for Predicting Burns in Surface Grinding of Steel. International Journal of Machine Tools and Manufacture, 44, 563571.

29. Marinescu, I. D., Hitchiner, M. P., Uhlmann, E., Rowe, W. B., \& Inasaki, I. (2006). Handbook of machining with grinding wheels. CRC Press. DOI: 10.1201/9781420017649.

30. Kikuchi, M. (2009). The Use of Cutting Temperature to Evaluate the Machinability of Titanium Alloys. Acta Biomaterialia, 5 (2), 770-775. DOI: 10.1016/j. actbio.2008.08.016

31. Nik, M. G., Movahhedy, M. R., \& Akbari, J. (2012). Ultrasonic-Assisted Grinding of Ti6Al4V Alloy. Procedia CIRP, 1, 353-358. DOI: 10.1016/j.procir.2012.04.063.

32. ISO (2019). DSTU ISO 603-3:2019. Communication Abrasives. Dimensions. Part 3. Internal grinding wheels. Amendment, 1. 